J. Lake Sci.(湖泊科学), 2009, 21(5): 637-646

http://www.jlakes.org. E-mail: jlakes@niglas.ac.cn

(C2009 by Journal of Lake Sciences

\title{
小白洋淀水一沉积物系统多环芳烃的分布、来源与生态风险*
}

\author{
朱 樱, 吴文婧, 王军军, 秦 宁, 王 印, 王 雁, 何玘霜, 徐福留**
}

(北京大学城市与环境学院, 地表过程分析与模拟教育部重点实验室, 北京 100871)

摘 要: 以端村小白洋淀为研究对象, 利用 GC-MS 测定了 6 个样点水、悬浮物和沉积物中 15 种优控多环芳烃(PAHs)的含量, 分析了其组成与来源特征, 探讨了不同多环芳烃单体的生态风险. 结果表明: (1)15 种优控多环芳烃的总含量(PAH15), 水相为 40.1-74.0ng/L, 算术均值 $51.0 \mathrm{ng} / \mathrm{L}$; 悬浮物为 $2438.0-5927.0 \mathrm{ng} / \mathrm{g}$, 算术均值 $4528 \mathrm{ng} / \mathrm{g}$; 沉积物为 $466.9-1366.4 \mathrm{ng} / \mathrm{g}$, 算术均值 为 $755.6 \mathrm{ng} / \mathrm{g}$; 与国内外有关研究相比, 污染较轻. (2)三相中均以 $2 、 3$ 环 PAHs 为主, 其比例均高于 $80 \%$; 并且, 从水相、悬浮 物相到沉积物相, 2 环 PAHs 依次降低, 3 环、4 环依次升高, 高环检出率和含量也依次升高. (3)沉积物中多环芳烃的来源以生 物质燃料(秸秆、薪材)和煤的燃烧为主, 以液体化石燃料(汽油、柴油和原油)的燃烧为辅. (4)沉积物中的芴(FLO)、菲(PHE)含 量在潜在生态风险效应区间低值(ERL)与中值(ERM)之间，其生态风险几率介于 $10 \%-50 \%$ 之间; 其他 PAHs 单体的含量均低于 ERL，其生态风险几率均低于 $10 \%$.

关键词: 小白洋淀; 多环芳烃; 分布; 组成; 来源; 生态风险

\section{Distribution, sources and ecological risks of polycyclic aromatic hydrocarbons in water-sediment system in Lake Small Baiyangdian}

ZHU Ying, WU Wenjing, WANG Junjun, QIN Ning, WANG Yin, WANG Yan, HE Qishuang \& XU Fuliu

(College of Urban \& Environmental Sciences, MOE Laboratory for Earth Surface Process, Peking University, Beijing 100871, P.R.China)

Abstract: The contents of 15 priority polycyclic aromatic hydrocarbons (PAHs) in water, suspended particulate matter (SPM) and sediment from six sampling sites in Lake Small Baiyangdian nearby Duancun Village were measured by GC-MS. The PAHs distribution, composition, source and ecological risk were analyzed. The following results were obtained: (1) the total contents of 15 priority PAHs (PAH15) varied from 40.1 to $74.0 \mathrm{ng} / \mathrm{L}$ with the average of $51.0 \mathrm{ng} / \mathrm{L}$ in water, from 2438.0 to $5927.0 \mathrm{ng} / \mathrm{g}$ with the average of $4528 \mathrm{ng} / \mathrm{g}$ in SPM, and from 466.9 to $1366.4 \mathrm{ng} / \mathrm{g}$ with the average of $755.6 \mathrm{ng} / \mathrm{g}$ in sediments. Compared with other areas in or out of China, the PAHs contamination was not serious. (2) The percentages of 2- and 3-rings PAHs accounted for more than $80 \%$ of PAH15 in water, SPM and sediment, respectively. From water, SPM, to sediments, the percentages of 2-rings PAHs were declined sequentially; while these of 3- and 4-rings as well as 5- and 6-rings PAHs were increased gradually. (3) The major sources of PAHs in sediments were the combustions of biomass fuels (straw, firewood) and coal; while the combustions of liquid fossil fuels including gasoline, diesel and crude oil was the secondary sources. (4) Owing to the contents of Fluorene (FLO) and Phenanthrene (PHE) varying between the ERL(effects range low) and ERM (effects range median), their probabilities of ecological risks was estimated between $10 \%-50 \%$. The probabilities of ecological risks for the rest species of 15 priority PAHs were below $10 \%$, since their contents were lower than the ERL.

Keywords: Lake Small Baiyangdian; PAHs; distribution; composition; source; ecological risk

* 国家杰出青年基金项目(40725004)、国家自然科学基金项目(40671165)和国家重点基础研究发展计划(973)项目 (2007CB407304, 2006CB403304)联合资助. 2009-02-03 收稿; 2009-03-30 收修改稿. 朱樱, 女, 1984 年生, 硕士研究生; E-mail: xiaoying8441@163.com.

** 通讯作者; E-mail: xufl@urban.pku.edu.cn. 

多环芳烃(PAHs, Polycyclic Aromatic Hydrocarbons)是一类由两个或两个以上苯环按线形、角形或簇 状等稠环方式相连组成的有机化合物，主要来源于有机物的不完全燃烧，如炼焦、工业和生活中煤炭/石 油/生物质燃烧、垃圾焚烧和交通排放等 ${ }^{[1-4]}$. PAHs 广泛分布于大气、颗粒物、土壤、水体、生物体等环 境介质中, 并可以在各环境介质间迁移转化. 天然水体是个复杂的生态系统, 包括水相、悬浮物相、沉积 物相以及各种生物相, 是 PAHs 存在的重要环境介质. PAHs 对生物体具有致癌、致畸、致突变的危害 ${ }^{[5]}$, 研 究其在水体中的组成、分布与来源对于评价水体 PAHs 污染状况及其生态风险具有重要意义. 国内外研 究表明, 地表水环境系统中均存在不同程度的 PAHs 污染 ${ }^{[6-15]}$.

白洋淀是我国华北地区最大的淡水湖泊，素有“华北明珠”之称，位于京、津、保三角地带，对 维持华北地区的生态环境起着举足轻重的作用; 同时, 白洋淀还承担着河北省保定与沧州两市淀区生 活、生产用水的重任. 随着周边以及上游地区社会经济的快速发展，排人白洋淀的工业废水与生活污水 不断增加; 另外, 淀区周围居民日常生活的煤、柴的燃烧以及交通运输、旅游与渔船燃油等排放的 PAHs, 也会通过大气干湿沉降进人白洋淀. 这些残留在水-沉积物系统中的 PAHs 会对水生生态系统甚至居民生 活产生不利影响. 本文主要研究小白洋淀水体、悬浮物、沉积物中 PAHs 残留水平与组成特征, 并探讨其 可能来源与生态风险，以期为白洋淀 PAHs 污染防治提供理论依据.

\section{1 材料与方法}

\section{1 样品采集}

由于受淀区地形影响，白洋淀被分割成大小不等、互相联系的 100 多个淀泊，主要有小白洋淀、马棚淀、 烧车淀、羊角淀、池鱼淀、后塘淀等. 本研究以位于端村以南、面积最大(约 $13.3 \mathrm{~km}^{2}$ )的小白洋淀为研究对 象, 布设了 6 个采样点, 于 2007 年 10 月 7 日采集了水与沉积物等样品, 样点采用 GPS 定位, 各采样点坐标 与环境状况如表 1 所示. 对于水深大于 $1 \mathrm{~m}$ 的采样点, 水样采取表层水(水面以下 $0-0.15 \mathrm{~m}$ )、中层水(水面以 下 $0.5-0.65 \mathrm{~m}$ )、底层水(沉积物以上 0-0.15m)混合采集的方式; 对于水深小于 $1 \mathrm{~m}$ 的采样点, 水样采取表层 水(水面以下 $0-0.15 \mathrm{~m}$ )和底层水(沉积物以上 $0-0.15 \mathrm{~m}$ )混合采集的方式; 将采集水样储存于棕色广口玻璃瓶 中; 表层沉积物用抓斗采泥器采集, 置于密封袋中保存. 所有样品均当天运回实验室.

表 1 小白洋淀采样点坐标与环境状况

Tab.1 The coordinates and surroundings of sampling stations in Lake Small Baiyangdian

\begin{tabular}{|c|c|c|c|c|c|}
\hline 样点 & 纬度 & 经度 & 水深 $(\mathrm{m})$ & 水温 $\left({ }^{\circ} \mathrm{C}\right)$ & 样点情况 \\
\hline 1 & $38^{\circ} 50^{\prime} 38^{\prime \prime} \mathrm{N}$ & $115^{\circ} 57^{\prime} 31^{\prime \prime E}$ & 0.7 & 15.5 & 水清澈见底, 沉水植物茂盛 \\
\hline 2 & $38^{\circ} 50^{\prime} 01^{\prime \prime} \mathrm{N}$ & $115^{\circ} 57^{\prime} 90^{\prime \prime} \mathrm{E}$ & 1.2 & 16 & 水稍浑, 有少量沉水植物 \\
\hline 3 & $38^{\circ} 49^{\prime} 45^{\prime \prime} \mathrm{N}$ & $115^{\circ} 57^{\prime} 35^{\prime \prime} \mathrm{E}$ & 1.9 & 16 & $\begin{array}{l}\text { 水清澈见底, 沉水植物茂盛, 岸上附 } \\
\text { 近有采油井 }\end{array}$ \\
\hline 4 & $38^{\circ} 50^{\prime} 51^{\prime \prime} \mathrm{N}$ & $115^{\circ} 55^{\prime} 94^{\prime \prime} \mathrm{E}$ & 0.8 & 16 & $\begin{array}{l}\text { 水较清澈, 沉水植物较为茂盛, 水面 } \\
\text { 有浮萍 }\end{array}$ \\
\hline 5 & $38^{\circ} 50^{\prime} 65^{\prime \prime} \mathrm{N}$ & $115^{\circ} 56^{\prime} 66^{\prime \prime} \mathrm{E}$ & 1.9 & 17 & 无沉水植物, 周围有养鱼网箱 \\
\hline 6 & $38^{\circ} 50^{\prime} 89^{\prime \prime} \mathrm{N}$ & $115^{\circ} 56^{\prime} 96^{\prime \prime} \mathrm{E}$ & 2.0 & 16 & $\begin{array}{l}\text { 靠近村庄, 无沉水植物, 周围有养鱼 } \\
\text { 网箱, 沉积物样有臭味 }\end{array}$ \\
\hline
\end{tabular}

\section{2 样品分析与测定}

将 $0.45 \mu \mathrm{m}$ 孔径玻璃纤维滤膜用 $450^{\circ} \mathrm{C}$ 高温灼烧 $4 \mathrm{~h}$, 恒重 $24 \mathrm{~h}$ 后称取空白滤膜重量. 将 $1 \mathrm{~L}$ 混合均匀 的水样用准备好的滤膜过滤，分别获得过滤后的水样和悬浮物. 过滤装置为 Millipore 公司的型号为 $80 \mathrm{EL} 005$ 的蠕动洜连接直径为 $142 \mathrm{~mm}$ 的过滤盘. 水样保存在棕色玻璃瓶中, 提取前加人多环芳烃回收率 指示物 NAP-d8、ACE-d10、ANT-d10、CHR-d12 和 Perylene-d12. 其多环芳烃用美国 Supelco 公司的 C18 固相萃取柱 $(6 \mathrm{ml}, 0.5 \mathrm{~g})$ 萃取, 用 $10 \mathrm{ml}$ 左右的二氯甲烷分三次洗脱, 洗脱液用无水硫酸钠柱除去水分后浓 缩至约 1-2ml, 加人 $15 \mathrm{ml}$ 正己烷替换溶剂, 旋转蒸发浓缩至约 $1 \mathrm{ml}$, 加人内标化合物(2-fluoro-1, 
1'-biphenyl 和 p-terphenyl-d14: J\&K Chemical, USA)待测. 注意，为了消除洗脱杂质的影响，应根据洗脱 液的颜色决定是否需要进一步净化, 如果洗脱液无色澄清, 并且在利用 GC-MS 试测时, 无明显杂质峰出 现, 各 PAHs 单体出峰较好, 可不需要进行净化; 但是, 如果发现洗脱液有颜色, 说明有杂质存在, 必需 进行净化. 本研究出现的是第一种情况.

将滤有悬浮物的滤膜冷冻干燥后称取重量, 减去之前称取的空白滤膜重量, 得到悬浮物重量. 将滤 膜剪碎，用净化的滤纸包好，加人多环芳烃回收率指示物(同上), 用索氏提取法提取. 在底瓶中加人 $100 \mathrm{ml}$ 二氯甲烷和正己烷的混合液 $(\mathrm{V}: \mathrm{V}, 1: 1)$ 以及活化过的铜粉. 水浴锅温度控制在 $55^{\circ} \mathrm{C}$ 左右, 索提约 $24 \mathrm{~h}$. 提取液用硅胶柱净化，加人 $20 \mathrm{ml}$ 正己烷淋洗饱和烷烃. 然后加人 $50 \mathrm{ml}$ 混合溶剂(正己烷:二氯甲烷，3:2) 洗脱 PAHs 组分. 含 PAHs 的洗脱液通过旋转蒸发, 浓缩至约 $1 \mathrm{ml}$ 加人内标化合物待测.

沉积物经冷冻干燥后研磨过 80 目耖. 称取经篮分混匀的样品 $10 \mathrm{~g}$, 用净化的滤纸包好, 加人多环芳 烃回收率指示物(同上), 用正已烷和二氯甲烷混合溶剂(V:V, 1:1)索氏提取 $24 \mathrm{~h}$. 提取液加人活化后的高纯 铜粉脱硫后, 在旋转蒸发仪上浓缩到 1-2ml, 加人约 $15 \mathrm{ml}$ 正已烷替换溶剂, 继续浓缩至约 $1 \mathrm{ml}$. 将浓缩后 的提取液通过硅胶层析柱净化, 其净化方法与悬浮物相同. 最后提取液经过浓缩, 加人内标化合物待测.

所有待测样品采用 GC-MS(Agilent GC6890/5973 MSD)分析测定 PAHs 组分. GC 条件: HP-5 MS $30 \mathrm{~m} \times 0.250 \mathrm{~mm}$ 毛细管色谱柱，载气为高纯 $\mathrm{He}$, 不分流进样, 进样量为 $1 \mu 1$, 初始柱温 $60^{\circ} \mathrm{C}$, 以 $5^{\circ} \mathrm{C} / \mathrm{min}$ 速 度升温至 $300^{\circ} \mathrm{C}$, 保留 $20 \mathrm{~min}$ 至样品完全流出. MSD 条件: $\mathrm{EI}$ 电离源 $70 \mathrm{eV}$, 质量范围 45-600amu, 倍增器 电压 $1288 \mathrm{~V}$, 离子源温度 $230^{\circ} \mathrm{C}$, 选择离子检测 $(\mathrm{SIM})$ 模式.

每一采样点样品设置 3 个平行样, 处理样品同时完成方法空白和程序空白. PAHs 在三相中的测定回 收率和检出限如表 2 所示. 使用 Microsoft Excel 和 SPSS 进行统计分析.

表 2 PAHs 测定方法回收率和检测限

Tab.2 Method recovery and detection limits of PAHs

\begin{tabular}{llllllllllllllllll}
\hline 化合物名称 & NAP & ACY ACE FLO & PHE & ANT FLA & PYR & BaA & CHR & BbF & BkF & BaP & IcdP & DahA BghiP \\
\hline 回收率(\%) & & & & & & & & & & & & & & & & & \\
水 & 35 & 96 & 98 & 90 & 96 & 99 & 102 & 105 & 99 & 76 & 102 & 74 & 76 & 31 & 21 & 40 \\
悬浮物 & 53 & 40 & 71 & 82 & 94 & 54 & 101 & 91 & 86 & 92 & 102 & 112 & 48 & 97 & 86 & 87 \\
沉积物 & 55 & 58 & 61 & 64 & 74 & 65 & 82 & 83 & 88 & 81 & 91 & 81 & 75 & 74 & 69 & 67 \\
方法检测限 & & & & & & & & & & & & & & & & \\
水(ng/L) & 59 & 4 & 5 & 24 & 40 & 3 & 4 & 6 & 2 & 2 & 2 & 2 & 2 & 2 & 2 & 2 \\
悬浮物(ng/g) & 70 & 3 & 3 & 11 & 32 & 3 & 6 & 3 & 2 & 2 & 2 & 2 & 2 & 2 & 2 & 2 \\
沉积物(ng/g) & 25 & 1 & 1 & 8 & 20 & 1 & 3 & 2 & 1 & 1 & 3 & 3 & 3 & 1 & 1 & 1 \\
\hline
\end{tabular}

\section{2 结果与讨论}

\section{$2.1 \mathrm{PAHs}$ 的分布与组成特征}

小白洋淀水、悬浮物与沉积物三相中 PAHs 的含量及其与国内外其他地区比较见表 3, 不同样点 PAHs 的含量与组成见图 1, PAHs 在三相中的百分组成见图 2 .

沉积物中 15 种 PAHs 均能检出, PAHs 总量为 $755.6 \pm 327.7 \mathrm{ng} / \mathrm{g}$; 水相中检出 9 种 PAHs 单体, 包括 6 种 2、3 环 PAHs 以及 3 种四环 PAHs((葱(ANT)、若葱(FLA)和萠(PYR)), 2 种四环 PAHs(苯并(b)若葱 $(\mathrm{BbF})$ 和苯并 $(\mathrm{k})$ 菼蒽 $(\mathrm{BkF})$ 以及 4 种 $5 、 6$ 环 PAHs 未检出, 检出的 PAHs 总量为 $51.04 \pm 11.88 \mathrm{ng} / \mathrm{L}$; 悬浮物中有 3 种 5、6 环 PAHs(狮并 $(1,2,3-c d)$ 萠(IcdP)、二苯并 $(a, h)$ 葱(DahA)和苯并 $(g h i)$ 荘(BghiP))未检出, 其他 2-4 环 PAHs 以及 5 环的苯并 (a)萠 $(\mathrm{BaP})$ 均能检出, 检出的 PAHs 总量为 $4528 \pm 1522 \mathrm{ng} / \mathrm{g}$, 约为沉积物中检出 PAHs 总量的 6 倍(表 3). 水、悬浮物与沉积物中均以低环的菲(PHE)和芴(FLO)含量最高. 水与悬浮物中 PAHs 平均含量低于大辽河流域、环渤海西部地区地表水、天津河流以及太湖的相应含量; 小白洋淀沉积物中 PAHs 平均含量高于大辽河流域与天津地区河流、斯里兰卡贝拉湖以及南意大利港沉积物，低于太湖、美 
国波士顿港以及澳大利亚悉尼港沉积物, 并且低于环渤海西部地区主要水体沉积物中平均含量.

水、悬浮物与沉积物中 PAHs 含量与组成存在空间差异. 6 个样点沉积物均检出五六环 PAHs, 其中样 点 6 沉积物中 PAHs 总含量以及五六环 PAHs 均最高, 其次为样点 3, 而样点 1 最低(图 1a). 6 个样点悬浮 物中均未检出五六环 PAHs, 并且样点 $1 、 2$ 悬浮物中四环 PAHs 也未检出; 样点 6 悬浮物中 PAHs 总含量 最高, 其次为样点 $1 、 4$, 而样点 3 最低(图 1b). 6 个样点水相中均未检出五六环 PAHs, 样点 3 水相中 PAHs

表 3 小白洋淀水、悬浮物与沉积物中 PAHs 含量及其与国内外其他地区比较

Tab.3 PAHs contents in water, SPM, sediments in Lake Small Baiyangdian and their comparisons with other regions in and out of China

\begin{tabular}{|c|c|c|c|c|c|c|}
\hline \multirow{2}{*}{ PAHs } & \multicolumn{2}{|c|}{ 水相 $(n g / L)$} & \multicolumn{2}{|c|}{ 悬浮物(ng/g) } & \multicolumn{2}{|c|}{ 沉积物(ng/g) } \\
\hline & 范围 & 均值土标准差 & 范围 & 均值士标准差 & 范围 & 均值土标准差 \\
\hline ACY & $3.2-5.0$ & $4.1 \pm 0.7$ & $37.8-77.1$ & $63.7 \pm 15.0$ & $1-3.3$ & $2.0 \pm 0.9$ \\
\hline $\mathrm{ACE}$ & $2.8-5.4$ & $3.7 \pm 0.9$ & $197-481$ & $324.3 \pm 101.3$ & $3.5-17.0$ & $8.3 \pm 4.7$ \\
\hline FLO & $16.8-26.9$ & $20.2 \pm 3.6$ & $666-1480$ & $1152.4 \pm 382.0$ & $94.7-271.5$ & $156.4 \pm 61.9$ \\
\hline PHE & $11.6-27.7$ & $16.7 \pm 5.6$ & $698.3-2728$ & $1642.4 \pm 731.8$ & $249.3-672.0$ & $398.4 \pm 155.1$ \\
\hline ANT & $0.2-1.4$ & $0.9 \pm 0.5$ & 92.9-2061 & $496.8 \pm 768.6$ & $8.9-40.0$ & $18.3 \pm 11.1$ \\
\hline FLA & $2.2-4.6$ & $3.0 \pm 0.9$ & $215.3-426.2$ & $337.8 \pm 84.0$ & $35.9-146.4$ & $73.4 \pm 42.6$ \\
\hline PYR & $1.1-2.7$ & $2.0 \pm 0.6$ & $117.3-270.6$ & $210.7 \pm 56.6$ & $22.8-82.4$ & $39.2 \pm 23.6$ \\
\hline $\mathrm{BaA}$ & $0-0.24$ & $0.1 \pm 0.1$ & $0-146.5$ & $46.7 \pm 55.1$ & $2.7-12.7$ & $5.2 \pm 3.9$ \\
\hline CHR & $0.2-0.8$ & $0.4 \pm 0.2$ & $88.0-451.5$ & $202.3 \pm 127.8$ & $8.3-35.1$ & $16.8 \pm 9.9$ \\
\hline $\mathrm{BbF}$ & - & - & $0-95.2$ & $25.2 \pm 36.5$ & $7.1-29.9$ & $13.6 \pm 8.6$ \\
\hline $\mathrm{BkF}$ & - & - & $0-109.4$ & $21.9 \pm 43.1$ & $2.9-11.3$ & $5.3 \pm 3.2$ \\
\hline $\mathrm{BaP}$ & - & - & $0-16.1$ & $3.9 \pm 6.7$ & $2.1-10.3$ & $4.4 \pm 3.1$ \\
\hline IcdP & - & - & - & - & $3.3-18.2$ & $7.0 \pm 5.7$ \\
\hline DahA & - & - & - & - & $0.6-3.1$ & $1.2 \pm 0.9$ \\
\hline BghiP & - & - & - & - & $2.8-13.5$ & $6.0 \pm 4.0$ \\
\hline $\mathrm{PAH}_{15}$ & $40.1-74.0$ & $51.0 \pm 11.9$ & $2438-5927$ & $4528 \pm 1522$ & $466.9-1366.4$ & $755.6 \pm 327.7$ \\
\hline $\begin{array}{l}\text { 白洋淀湿地 } \\
\text { 表层沉积物 }^{[6]}\end{array}$ & - & - & - & - & $324.6-1738.5$ & $588.8 \pm 219.3$ \\
\hline 大辽河流域 ${ }^{[7]}$ & $946.1-13448.5$ & 6471.1 & $317.5-238518.7$ & 21724.5 & $61.9-840.5$ & 287.3 \\
\hline $\begin{array}{l}\text { 环渤海西部 } \\
\text { 地区地表水 }{ }^{[8]}\end{array}$ & 7.4-10174.2 & $432 \pm 1568$ & $43.0-34024.1$ & $4538.7 \pm 5962.6$ & $2.4-27153.0$ & $1029.0 \pm 4043.1$ \\
\hline $\begin{array}{l}\text { 天津河流水- } \\
\text { 沉积物系统 }^{[9]}\end{array}$ & $45.8-1271.6$ & $281.6 \pm 336.9$ & $938.3-64189.4$ & $13516.8 \pm 14199.7$ & $0.8-531.5$ & $46.0 \pm 105.2$ \\
\hline$太^{\text {太湖 }}{ }^{[10]}$ & $37.5-183.5$ & $134.5 \pm 54.8$ & $3369.6-7531.1$ & $5607.6 \pm 1575.9$ & 2091.8-4094.4 & $3156.1 \pm 814.7$ \\
\hline $\begin{array}{l}\text { 美国波士顿 } \\
\text { 港沉积物 }\end{array}$ & - & - & - & - & $7300-358000$ & - \\
\hline $\begin{array}{l}\text { 斯里兰卡贝 } \\
\text { 拉湖 }{ }^{[12]}\end{array}$ & & & & & $152-569$ & 329 \\
\hline $\begin{array}{l}\text { 南意大利港 }{ }^{[13]} \\
\text { 澳大利亚悉 } \\
\text { 尼港 }^{[14]}\end{array}$ & & & & & $\begin{array}{l}9-31774 \\
<100-380000\end{array}$ & 3142 \\
\hline $\begin{array}{l}\text { 土耳其马尔马拉 } \\
\text { 海 Gemlik 湾 } \\
\text { [15] }\end{array}$ & & & & & $50.8-13482$ & \\
\hline
\end{tabular}



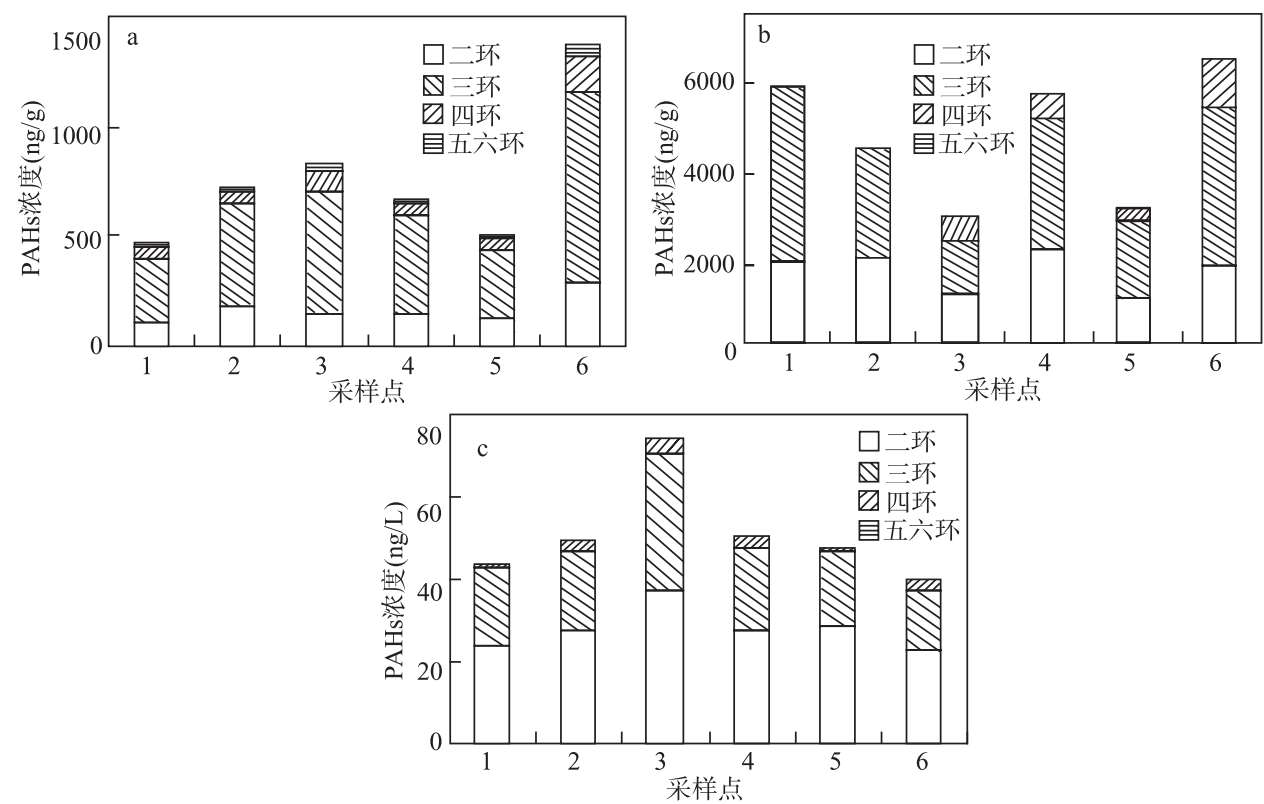

图 1 小白洋淀三相中多环芳烃含量与组成(a: 沉积物; b: 悬浮物; c: 水)

Fig.1 PAHs contents and composition in Small Baiyangdian Lake(a: sediments; b: SPM; c: water)

总含量最高, 其他 5 个采样点含量相近. 这些差别 可能与样点所处的环境条件有关. 样点 6 靠近居民 点和旅游码头, 并且其附近有多处围网养鱼的水面, 可能造成样点 6 悬浮物与沉积物中 PAHs 含量较高; 样点 3 附近的地面上有一口正在采油的油井, 可能 会有石油类物质泄漏至湖水中.

三相中 2 环和 3 环 PAHs 均占到 $80 \%$ 以上; 水 相和悬浮物相均未测到高环 PAHs, 仅有沉积物相 测到五六环 PAHs，占 $2.5 \%$; 从水相、悬浮物相到沉 积物相, 低环和中环 PAHs 含量百分比依次降低, 中高环依次升高. 2 环 PAHs 百分含量在水相中最高, 为 $54.9 \%$, 悬浮物和沉积物中依次减少, 沉积物中 2 环 PAHs 仅占 $22.1 \%$. 3 环 PAHs 百分含量在沉积物 中最高, 为 $64.9 \%$, 悬浮物和水相中依次减少, 水相 中 3 环 PAHs 仅占 $40.3 \%$ (图 2).

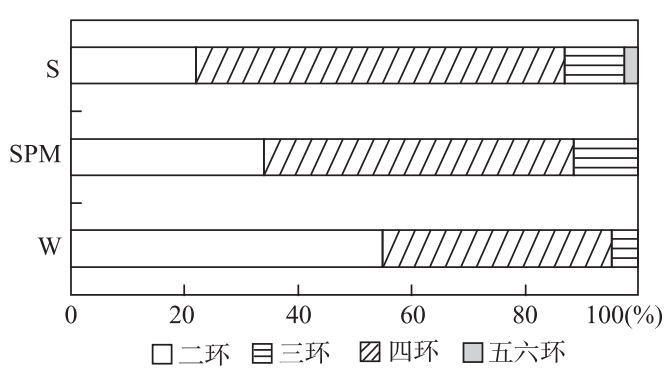

图 2 小白洋淀水 $(\mathrm{W})$ 、悬浮物 $(\mathrm{SPM})$ 与 沉积物(S)中 PAHs 的平均百分组成

Fig. 2 The average percentage of different ring PAHs in water(W), suspended particulate matter(SPM) and sediment (S) in Lake Small Baiyangdian

\subsection{PAHs 在三相中的分配}

将三相中 PAHs 浓度进行对数变换, 经检验符合对数正态分布; 这符合环境中多数污染物呈对数正 态分布的统计特征 ${ }^{[16]}$. 利用 Pearson 相关性分析发现, 在 0.05 显著水平下, 水相和悬浮物相中的 PAHs 含 量呈显著相关; 而水相和沉积物相、悬浮物相和沉积物相中 PAHs 含量的相关性不显著.

有机碳吸附平衡常数 $K_{\mathrm{oc}}$ 可以定量描述污染物质在固相和水相间的分配情况. 如果水中的 PAHs 在悬 浮颗粒物(SPM) 和水相(Water)之间达到稳态分配平衡, 则可计算颗粒物/水分配常数 $K_{\mathrm{p}}^{\mathrm{obs}}$ 以及有机碳吸附 平衡常数 $K_{\mathrm{oc}}{ }^{\mathrm{obs}}$. PAHs 在小白洋淀水、悬浮物、沉积物三相间有机碳吸附平衡常数的计算结果(表 4)表明, 小白洋淀的悬浮物/水、沉积物/水分配系数均高于文献预测值. 这与 Foster 等 ${ }^{[17]}$ 对 Chesapeake 海湾悬浮 物/水的报道, 以及 McGroddy ${ }^{[18-19]}$ 对 Boston 港的沉积物研究结果一致. 其可能原因是, 燃烧裂解产生的 
PAHs 吸附并被锁定在煤烟和飞灰颗粒物上，对于一般吸附解析过程无效 ${ }^{[17,19]}$.

表 4 PAHs 在水、悬浮物、沉积物三相间有机碳吸附平衡常数*

Tab.4 The organic carbon adsorption equilibrium constant of PAHs among water, SPM, sediments

\begin{tabular}{|c|c|c|c|c|c|c|}
\hline \multirow[b]{2}{*}{ PAHs } & \multirow{2}{*}{$\begin{array}{l}\text { 文献值 } \\
\lg K_{\mathrm{oc}}{ }^{\text {pre }}\end{array}$} & \multicolumn{2}{|c|}{ 悬浮物/水 } & \multicolumn{2}{|c|}{ 沉积物/水 } & \multirow{2}{*}{$\begin{array}{c}\text { 悬浮物/沉积物 } \\
K_{\mathrm{oc} \text { 水沉沉积物 }} / \\
K_{\mathrm{oc}} \text { 水_悬浮物 } \\
\end{array}$} \\
\hline & & $\lg K_{\mathrm{oc}}^{\mathrm{obs}}$ & $\begin{array}{c}\lg K_{\mathrm{oc}}{ }^{\text {obs }} \\
-\lg K_{\mathrm{oc}}{ }^{\text {pre }}\end{array}$ & $\lg K_{\mathrm{oc}}^{\mathrm{obs}}$ & $\begin{array}{c}\lg K_{\mathrm{oc}}{ }^{\text {obs }} \\
-\lg K_{\mathrm{oc}}{ }^{\text {pre }} \\
\end{array}$ & \\
\hline $\mathrm{ACY}$ & 3.68 & 4.54 & 0.86 & 4.23 & 0.55 & 0.49 \\
\hline $\mathrm{ACE}$ & 3.60 & 5.29 & 1.69 & 4.89 & 1.29 & 0.39 \\
\hline FLO & 3.86 & 5.11 & 1.25 & 5.43 & 1.57 & 2.07 \\
\hline PHE & 4.25 & 5.35 & 1.10 & 5.92 & 1.67 & 3.70 \\
\hline ANT & 4.22 & 6.11 & 1.89 & 5.86 & 1.64 & 0.56 \\
\hline FLA & 4.90 & 5.40 & 0.50 & 5.93 & 1.03 & 3.32 \\
\hline PYR & 4.86 & 5.37 & 0.51 & 5.83 & 0.97 & 2.84 \\
\hline $\mathrm{BaA}$ & 5.59 & 6.17 & 0.58 & 6.40 & 0.81 & 1.69 \\
\hline CHR & 5.54 & 6.10 & 0.56 & 6.21 & 0.67 & 1.27 \\
\hline
\end{tabular}

* 颗粒物/水分配常数 $K_{\mathrm{p}}{ }^{\mathrm{obs}}=[P A H s]_{\mathrm{SPM}} /[P A H s]_{\mathrm{water}}(1 / \mathrm{kg}),[P A H s]_{\mathrm{SPM}}$ 为悬浮物中 PAHs 的总浓度, $[P A H s]_{\mathrm{water}}$ 为水中 PAHs 的总浓度. 将 $K_{\mathrm{p}}{ }^{\mathrm{obs}}$ 用有机碳含量标准化, 得到有机碳吸附平衡常数 $\left(K_{\mathrm{oc}}{ }^{\mathrm{obs}}\right)$ 值: $K_{\mathrm{oc}}{ }^{\mathrm{obs}}=K_{\mathrm{p}}{ }^{\mathrm{obs}} / f_{\text {ocSPM}}$.

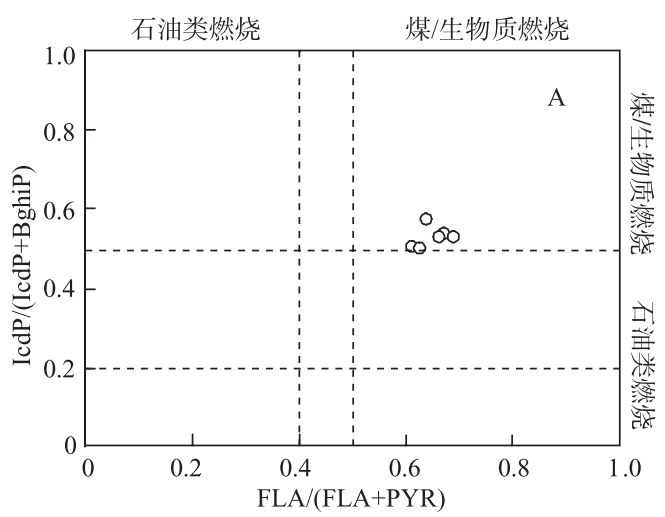

图 3 小白洋淀沉积物中多环芳烃来源诊断

Fig.3 Diagnostic ratios for source

identification in surface sediments from Lake Small Baiyangdian

\section{3 表层沉积物 PAHs 的可能来源}

Yunker 等对 FLA/(FLA+PYR)、IcdP/(IcdP+ BghiP)、ANT/(ANT+PHE)、BaA/(BaA+CHR)等常 用的 PAHs 同分异构体的特征比值的判定依据 ${ }^{[20-21]}$ 进行了归纳与总结, 认为 FLA 与 PYR、 IcdP 与 BghiP 的环境行为特征类似, 其比值在环境过程中 相对稳定，因此 FLA/(FLA+PYR)、IcdP/(IcdP+BghiP) 两组比值可信度较高 ${ }^{[22-23]}$. 由于 IcdP 和 BghiP 在水 和悬浮物中未检出, 因此仅用这两个比值分析沉积 物中 PAHs 的来源. 当 FLA/(FLA+PYR)小于 0.4 时 是石油源, 介于 0.4-0.5 之间时为源自液体化石燃 料(汽油、煤油、原油等)燃烧，大于 0.5 时源自草、 木材、煤的燃烧; 当 IcdP/(IcdP+BghiP)小于 0.2 时为 石油源, 介于 0.2-0.5 之间时源自液体化石燃料(汽 油、煤油、原油等)燃烧，大于 0.5 时源自草、木材、 煤的燃烧 ${ }^{[22-23]}$.

小白洋淀各采样点表层沉积物中 PAHs 的特征比值(图 3)表明，除样点 5 的 IcdP/(IcdP+BghiP)略小于 0.5 外, 其余各样点的两组特征比值均大于 0.5 , 说明 PAHs 主要来自草、木材、煤的燃烧; 另外, 由于 $\mathrm{IcdP} /(\mathrm{IcdP}+\mathrm{BghiP})$ 只是稍高于 0.5 , 在图 4 中靠近于混合区, 表明沉积物中 PAHs 可能受液体化石燃料(汽 油、煤油、原油等)燃烧的影响. 这与淀区居民生活方式与燃料结构相符, 并且反映了水上旅游机动船以 及陆上交通的一定影响. 这也印证了 2.2 节中有机碳吸附平衡常数的分析结果.

上述比值法只能粗略判定研究区内的 PAHs 来源. 为了更准确的判断小白洋淀沉积物中 PAHs 的来源, 本研究利用 SPSS 软件对 6 个采样点的沉积物 PAHs 数据进行因子分析; 虽然本研究样本量较少, 但经 $\mathrm{KMO}$ 检验结果为 0.697 , 大于 0.5 , 适合做因子分析 ${ }^{[24]}$. 采用主成分分析法结合方差极大正交旋转, 提 
取累计方差贡献率大于 $90 \%$ 的因子(表 5). 主因 子 1 主要由中、高环 PAHs 以及个别低环 PAHs 构成, 按得分的高低, 分别为: 范 $(\mathrm{ACY})$ 、苯并 $[\mathrm{g}$, $h, i]$ 萠 $(B g h i P) 、$ 苯并 $[k]$ 荧葱 $(B k F) 、$ 、苯并 $(a h)$ 葸 $(\mathrm{DahA}) 、$ 苯并 $[\mathrm{a}]$ 萠 $(\mathrm{BaP}) 、$ 苯并 $[\mathrm{b}]$ 菼葱 $(\mathrm{BbF})$ 、 狮并 $(1,2,3-c d)$ 芘 $(\mathrm{IcdP})$ 、屈 $(\mathrm{CHR}) 、$ 苯并 $[a]$ 葱 $(\mathrm{BaA}) 、$ 葱(ANT)、萠(PYR); 主因子 2 主要由低 环 PAHs 构成, 按得分的高低, 分别为: 二氢苍 (ACE)、菲(PHE)、䓄(FLO)和菼葱(FLA)(表 5). 由 于不同燃烧源产生不同的特征化合物, 因此, 可 以根据 15 种组分的因子载荷结果来判断 PAHs 的来源. ACY 和 PHE 是木材/薪柴燃烧排放的优 势物种 ${ }^{[25-26]}$, BghiP 和 DahA 为交通汽油燃烧排 放的特征化合物 ${ }^{27-28]}$, PHE、ANT、FLA、PYR、 $\mathrm{CHR} 、 \mathrm{BkF}$ 和 $\mathrm{BbF}$ 为燃煤排放的主要 $\mathrm{PAHs}{ }^{[28-29]}$, $\mathrm{BkF} 、 \mathrm{BbF}$ 和 $\mathrm{CHR}$ 是中国家用燃煤排放的最主 要的中高环 $\mathrm{PAHs}^{[30-31]}$, IcdP 和 $\mathrm{BaA}$ 分别为交通 柴油燃烧与天然气燃烧排放的特征化合物 ${ }^{[28]}$, $\mathrm{BaP} 、 \mathrm{FLO}$ 和 ACE 被认为是炼焦排放的特征化 合物 ${ }^{[28,32]}$, FLA 和 PYR 是秸秆燃烧排放的最主 要的 PAHs 物种 ${ }^{[25]}$. 据此, 可以推断小白洋淀沉 积物中 PAHs 主要来源于木材/薪柴、煤、天然 气以及秥秆的燃烧、交通汽油和柴油的燃烧、以 及焦炉的炼焦生产(表 5).

\section{$2.4 \mathrm{PAHs}$ 的生态风险}

一般低环 (二、三环)的 PAHs 可以呈现显著的 急性毒性, 某些高分子量的 PAHs 具有潜在生态 效应 ${ }^{[33-34]}$. 中华人民共和国国家标准规定的地表 水环境质量标准规定苯并 (a) 芘 $(\mathrm{BaP})$ 标准值为 $2.8 \mathrm{ng} / \mathrm{L}^{[35]}$, 海水水质标准规定 $\mathrm{BaP}$ 应当不大于 $2.5 \mathrm{ng} / \mathrm{L}^{[36]}$. 本研究未检测到水相中的 BaP, 故其生态风险很小.

虽然在沉积物污染生态风险评价反面做了很多研究工作, 但仍未建立起统一的评价标准 ${ }^{[37]}$. Long 等 在大量实验研究的基础上提出用于确定河口、海洋沉积物中有机污染物的潜在生态风险的效应区间低值 (Effects Range Low, ERL)和效应区间中值(Effects Range Median, ERM), 并被视为反映沉积物质量的生态 风险水平 ${ }^{[38-39]}$. 当污染物浓度 $<$ ERL 时, 生物有害效应几率 $<10 \%$; 当污染物浓度 $>$ ERM 时, 生物有害效应 几率 $>50 \%$; 当污染物浓度在 ERL-ERM 之间时, 生物有害效应几率介于 $10 \%-50 \%$ 之间.

本文利用课题组前期研究使用的方法 ${ }^{[00]}$, 即用超标率和超标倍数定量评价各样点的风险情况. 其计 算公式如下:

$$
\begin{gathered}
M=C / \mathrm{ERL} \\
R=100 \times(C-\mathrm{ERL}) / \mathrm{ERL}
\end{gathered}
$$

式中, $M$ 为超标倍数; $C$ 为本研究多环芳烃测定浓度; $R$ 为超标率. 根据公式计算结果如表 6 所示.

沉积物中 FLO、PHE 在 6 个样点的浓度都超过 ERL, 介于 ERL 与 ERM 之间, 超标率分别达到 $398.5 \%-1329 \%$ 和 3.9\%-180\%; 而 ACE 超过 ERL 的样点数为 1 个, 超标率为 $6 \%$; 其他 PAHs 单体的浓度 均低于 ERL. 因此, FLO 和 PHE 对小白洋淀水生植物的生态风险几率为 $10 \%-50 \%$, ACE 对小白洋淀局部 
地区的水生生物的生态风险几率小于 $10 \%$ ，其他 PAHs 单体的生态风险很小(表 6).

表 6 小白洋淀沉积物 PAHs 残留量与 ERL 和 ERM 值的比较

Tab.6 Comparisons of PAHs residues in surface sediments from Lake Small Baiyangdian with ERL and ERM

\begin{tabular}{cccccccc}
\hline PAHs & ERL & ERM & 本研究 $(\mathrm{ng} / \mathrm{g})$ & 超标率 & 超标倍数 & $\begin{array}{c}<\text { ERL } \\
\text { 样点数 }\end{array}$ & $\begin{array}{c}>\text { ERM } \\
\text { 样点数 }\end{array}$ \\
\hline ACY & 44 & 640 & $1.0-3.2$ & 0 & 0 & 6 & 0 \\
ACE & 16 & 500 & $3.4-10.1$ & $6.0 \%$ & 1.1 & 5 & 0 \\
FLO & 19 & 540 & $94.7-271.5$ & $398.5 \%-1329 \%$ & $5.0-14.3$ & 0 & 0 \\
PHE & 240 & 1500 & $249.3-672.0$ & $3.9 \%-180 \%$ & $1.0-2.8$ & 0 & 0 \\
ANT & 85.3 & 1100 & $8.9-40.0$ & 0 & 0 & 6 & 0 \\
FLA & 600 & 5100 & $35.9-146.4$ & 0 & 0 & 6 & 0 \\
PYR & 665 & 2600 & $22.8-82.4$ & 0 & 0 & 6 & 0 \\
BaA & 261 & 1600 & $2.7-12.7$ & 0 & 0 & 6 & 0 \\
BaP & 430 & 1600 & $2.1-10.3$ & 0 & 0 & 6 & 0 \\
DahA & 63.4 & 260 & $0.6-3.1$ & 0 & 0 & 6 & 0 \\
\hline
\end{tabular}

\section{3 结论}

(1)三相中均以 2 环和 3 环 PAHs 含量最高, 达到 $80 \%$ 以上; 并且从水相、悬浮物相到沉积物相, 2 环 PAHs 依次降低, 3 环、4 环和高环依次升高. 小白洋淀水体污染程度与其他研究比较并不严重.

(2)小白洋淀实际测定的悬浮物/水、沉积物/水有机碳吸附平衡常数 $K_{\mathrm{oc}}{ }^{\mathrm{obs}}$ 均高于文献预测值; 并且多 数 PAHs 的 $K_{\mathrm{oc}}$ 水-沉积物大于 $K_{\mathrm{oc}}$ 水-悬浮物, 说明这些 PAHs 在沉积物上吸附比较紧密.

(3)小白洋淀沉积物中 PAHs 来源包括木材/薪柴、煤、天然气以及秸秆的燃烧、交通汽油和柴油的 燃烧、以及焦炉的炼焦生产; 其中以生物质燃料(秸秆、薪材)和煤的燃烧为主, 以液体化石燃料(汽油、 柴油和原油)的燃烧以及焦炉的炼焦生产为辅.

(4)水相中 PAHs 的生态风险很小; 沉积物中的芴(FLO)、菲(PHE)的生态风险几率介于 $10 \%-50 \%$ 之 间，其他 PAHs 单体的生态风险几率均低于 $10 \%$.

\section{4 参考文献}

[1] Baek SO, Field RA, Goldstone ME et al. A review of atmospheric polycyclic aromatic hydrocarbons: source, fate and behavior. Water Air and Soil Pollution, 1991, 60: 279-300.

[2] Khalili NR, Scheff PA, Holsen TM. PAH source fingerprints for coke ovens, diesel and gasoline engines, highway tunnels, and wood combustion emissions. Atmospheric Environment, 1995, 29: 533-542.

[3] Harrison RM, Smith DJT, Luhana L. Source apportionment of atmospheric polycyclic aromatic hydrocarbons collected from an urban location in Birmingham, U. K. Environmental Science and Technology, 1996, 30: 825-832.

[4] Xu SS, Liu WX, Tao S. Emission of polycyclic aromatic hydrocarbons in China. Environmental Science and Technology, 2006, 40: $702-708$

[5] IARC monographs on the evaluation of the carcinogenic risk of chemicals to humans. Overall evaluation of carcinogenity: an updating of IARC monographs volumes 1-42. Suppl. 7. International Agency for Research on Cancer, Lyon, France. 1987.

[6] 周怀东, 赵 健, 陆 瑾等. 白洋淀湿地表层沉积物多环芳烃的分布、来源及生态风险评价. 生态毒理学报, 2008, 3: 291-299.

[7] Guo W, He MC, Yang ZF et al. Distribution of polycyclic aromatic hydrocarbons in water, suspended particulate matter and sediment from Daliao River watershed, China. Chemosphere, 2007, 68: 93-104.

[8] 摆亚军. 环渤海西部地区地表水多环芳烃污染特征[学位论文]. 北京: 北京大学, 2008. 
[9] 施 治. 天津地区河流系统中的多环芳烃污染[学位论文]. 北京: 北京大学, 2003.

[10] Qiao M, Huang SB, Wang ZJ. Partitioning characteristics of PAHs between sediment and water in a shallow lake. Aquatic Ecosystems, 2008, 8(2): 69-73.

[11] Wang XC, Zhang YX, Chen RF. Distribution and partitioning of polycyclic aromatic hydrocarbons (PAHs) in different size fractions in sediments from Boston Harbor. Marine Pollution Bulletin, 2001, 42(11): 1139-1149.

[12] Pathiratne KAS, De Silva OCP, David H et al. Occurrence and distribution of polycyclic aromatic hydrocarbons (PAHs) in Bolgoda and Beira Lakes, Sri Lanka. Bull Environ Contam Toxicol, 2007, 79: 135-140.

[13] Sprovieri M, Feo ML, Prevedello L et al. Heavy metals, polycyclic aromatic hydrocarbons and polychlorinated biphenyls in surface sediments of the Naples harbour (southern Italy). Chemosphere, 2007, 67(5): 998-1009.

[14] McCready S, Slee DJ, Birch GF et al. The distribution of polycyclic aromatic hydrocarbons in surficial sediments of Sydney Harbour, Australia. Marine Pollution Bulletin, 2000, 40(11): 999-1006.

[15] Unlu S, Alpar B. Distribution and sources of hydrocarbons in surface sediments of Gemlik Bay (Marmara Sea, Turkey). Chemosphere, 2006, 64(5): 764-777.

[16] 陶 澍编. 应用数理统计方法. 北京: 中国环境科学出版社, 1994: 33 .

[17] Foster GD, Roberts EC, Gruessner B et al. Hydrogeochemistry and transport of organic contaminants in an urban watershed of Chesapeake Bay (USA). Applied Geochemistry, 2000, 15(7): 901-915.

[18] McGroddy SE, Farrington JW, Gschwend PM. Comparison of the in situ and desorption sediment-water partitioning of polycyclic aromatic hydrocarbons and polychlorinated biphenyls. Environmental Science \& Technology, 1996, 30(1): 172-177.

[19] McGroddy SE, Farrington JW. Sediment porewater partitioning of polycyclic aromatic hydrocarbons in three cores from Boston harbor, Massachusetts. Environmental Science \& Technology, 1995, 29: 1542-1550.

[20] Luthy RG, Aiken GR, Brusseau ML et al. Sequestration of hydrophobic organic contaminants by geosorbents. Environmental Science \& Technology, 1997, 31(12): 3341-3347.

[21] Zhou JL, Fileman TW, Evans S et al. Fluoranthene and pyrene in the suspended particulate matter and surface sediments of the Humber estuary, UK. Marine Pollution Bulletin, 1998, 36(8).

[22] Yunker MB, Macdonald RW, Vingarzan R et al. PAHs in the Fraser River basin: a critical appraisal PAH ratios as indicators of PAH source and composition. Organic Geochemistry, 2002, 33: 489-515.

[23] Zhang YL, Tao S, Liu WX et al. Source diagnostics of polycyclic aromatic hydrocarbons based on species ratios: a multimedia approach. Environmental Science and Technology, 2005, 39: 9109-9114.

[24] 罗应婷, 杨玉娟编著. SPSS 统计分析从基础到实践. 北京: 电子工业出版社, 2007: 293.

[25] Jenkins BM, Jones AD, Turn SQ et al. Emission factors for polycyclic aromatic hydrocarbons from biomass burning. Environmental Sciences and Technology, 1996, 30: 2462-2469.

[26] Lee RGM, Coleman P, Jones JL et al. Emission factors and importance of PCDD/Fs, PCBs, PCNs, PAHs, and PM10 from the domestic burning of coal and wood in the U.K. Environmental Sciences and Technology, 2005, 39: 1436-1447.

[27] Lim LH, Harrison RM, Harrad S et al. The contribution of traffic to atmospheric concentrations of polycyclic aromatic hydrocarbons. Environmental Sciences and Technology, 1999, 33: 3538-3542.

[28] Simcik MF, Eisenreich SJ, Lioy PJ. Source apportionment and source/sink relationships of PAHs in the coastal atmosphere of Chicago and Lake Michigan. Atmospheric Environment, 1999, 33: 5071-5079.

[29] Harrison RM, Smith DJT, Luhana L. Source apportionment of atmospheric polycyclic aromatic hydrocarbons collected from an urban location in Birmingham, U.K. Environmental Sciences and Technology, 1996, 30: 832-852.

[30] Chen YJ, Sheng GY, Bi XH et al. Emission factors for carbonaceous particles and polycyclic aromatic hydrocarbons residential coal combustion in China. Environmental Sciences and Technology, 2005, 39: 1861-1867.

[31] 窦 晗, 常 彪, 魏志成等. 国内民用燃煤烟气中多环芳烃排放因子研究. 环境科学学报, 2007, 27: 1783-1788.

[32] Khalili NR, Scheff PA, Holsen TM. PAH source fingerprints for coke ovens, diesel and gasoline engines, highway tunnels, and wood combustion emissions. Atmospheric Environment, 1995, 29: 533-542. 
[33] Douben PET. PAHs: an ecotoxicological perspective. New York: Wiley, 2003: 377.

[34] Witt G. Polycyclic aromatic hydrocarbons in water and sediment of the Baltic Sea. Mar Pollut Bull, 1995, 31(4-12): 237-248.

[35] GB 3838-2002. 中华人民共和国地表水环境质量标准, 2002.

[36] GB 3097-1997. 海水水质标准, 1997.

[37] McCauley DJ, DeGrave GM, L inton TK. Sediment quality guidelines and assessment: over and research needs. Environmental Sciences and Policy, 2000, 3: 133-144.

[38] Long ER, Macdonald DD, Smith SL et al. Incidence of adverse biological effects with ranges of chemical concentrations in marine and estuarine sediments. Environ Manage, 1995, 19(1): 81-97.

[39] Long ED, Field LJ, Macdonald DD. Predicting toxicity marine sediments with numerical sediment quality guidelines. Environ Toxicol Chem, 1998, 17(4): 714-727.

[40] 张 颖, 摆亚军, 徐福留等. 河北水库及湖泊沉积物中 DDT 农药的残留特征与风险评估. 环境科学学报, 2006, 26(4): 623-631. 